\title{
Scratch. Estrategia didáctica para el aprendizaje de las tablas de multiplicar en escuela nueva
}

\author{
Scratch. Didactic strategy to learn the \\ multiplication table in new school
}

Fecha de recepción: 15 de Mayo de 2016.

Fecha de aprobación: 15 de Noviembre de 2016.

Carlos Julio Vivas Silva* Zulma Lucía Murillo Chiquillo**

Artículo de Reflexión

\section{Resumen}

Al hacer un análisis del Proyecto Educativo Institucional, los resultados de las pruebas Saber de matemáticas, Plan de Estudios, Índice Sintético de Calidad Educativa, Modelo Escuela Nueva, metodología y apropiación del sistema educativo en la Institución, se ve la necesidad de fortalecer procesos multiplicativos y favorecer el pensamiento numérico en la formación integral del educando; reforzar el aprendizaje en la resolución de operaciones básicas y contribuir a desarrollar habilidades que respondan con eficiencia a solucionar problemas matemáticos de la vida cotidiana. El objetivo fue implementar una estrategia didáctica para facilitar el aprendizaje de las tablas de multiplicar con el uso de Scratch. La metodología tiene enfoque cualitativo, tipo de investigación acción educativa. Para ello, se realizó un diagnóstico para identificar debilidades y fortalezas de los estudiantes, se diseñaron actividades micro-curriculares, lúdico-
"Institución Educativa Juan José Rondón Tasco Boyacá, Colombia supervivas9@yahoo.es

**Juan José Rondón. Tasco - Boyacá, Colombia zulmalumu@yahoo.es

***Universidad Pedagógica y Tecnológica de Colombia - Boyacá, Colombia jaime.cristancho@uptc. edu.co

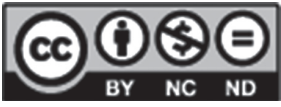


didácticas que fortalezcan los rincones de aprendizaje, dando al estudiante la oportunidad de disfrutar de ambientes de clase, pensar, sentir y hacer; siendo sujeto activo, dando iniciativas en su autoaprendizaje, estimulando la comprensión de procedimientos, generado espacios para el cálculo y descubrimiento. Como resultados, se

\section{Abstract}

When making an analysis of the Institutional Educational Project, the results of the SABER tests on Mathematics, Curriculum, Synthetic Index of Educational Quality, New School Model, methodology and appropriation of the educational system in the Institution, we see the need to strengthen multiplicative processes and favouring numerical thinking in the integral education of the student; reinforce learning in the resolution of basic operations and help develop skills that respond efficiently to solve mathematical problems of daily life. The objective was to implement a didactic strategy to facilitate the learning of the multiplication tables with the use of Scratch methodology. The methodology has a qualitative approach, type of educational action evidenció con el uso del software que los estudiantes lograron avances en el proceso de aprendizaje mejorando la capacidad de razonamiento de las tablas de multiplicar.

Palabras clave: tablas de multiplicar, software libre scratch, estrategia didáctica, Escuela Nueva.

research. For this, a diagnosis was made, identifying weaknesses and strengths of the students. micro-curricular and didactic activities were designed to strengthen the learning corners, giving the student the opportunity to enjoy class environments, think, feel and do; being an active subject, giving initiatives in their self-learning, stimulating the understanding of procedures, generating spaces for calculation and discovery. As results, it was evidenced with the use of software that the students achieved advances in the learning process by improving the reasoning capacity of the multiplication tables.

Keywords: multiplication tables, scratch free software, didactic strategy, Escuela Nueva methodology. 


\section{Introducción}

Los métodos de enseñanza han evolucionado como la tecnología; dando oportunidad de encontrar otras formas de aprendizaje; según las necesidades e intereses de la actualidad, un software libre usado en el aula permite crear escenarios divertidos para los estudiantes y tiene una temática educativa que fortalece los ambientes de aprendizajes, en este caso las tablas de multiplicar.

Las TIC se han convertido en herramientas lúdicas en el momento de afianzar los conocimientos, desarrollando actividades de observación, manipulación, clasificación, comparación, experimentación. El software libre scratch vivencia el aprendizaje significativo, convirtiéndose en una ayuda educativa, generando estrategias funcionales, contribuyendo a mejorar la calidad de vida de los estudiantes, generando espacios agradables para la adquisición del conocimiento. "Piaget plantea que se deben promover experiencias concretas que lleven al estudiante al conocimiento de hechos prácticos, no solo verbales" (Fundación Escuela Nueva, 2009, p. 183).

Se puede establecer, por medio de observación directa y participativa, que los estudiantes de la institución tienen problemas con el aprendizaje de las tablas de multiplicar, por ello, se hace necesario usar una estrategia didáctica que permita el aprendizaje de las tablas, para adquirir conocimientos a través de la ejercitación; integrando teoría y habilidades, que los lleve a resolver problemas matemáticos que requieran de la multiplicación, lo cual se busca lograr con esta propuesta.
Por consiguiente, se espera contribuir al aprendizaje de las tablas de multiplicar de manera lúdica, donde el estudiante consiga un aprendizaje placentero, significativo y explote, de manera adecuada, la capacidad de razonamiento matemático haciendo uso del software. Además, la propuesta planteada pretende dejar una puerta abierta para docentes de las zonas rurales, que sirva de ayuda educativa en los centros de recursos de aprendizaje (CRA), para que la adopten como una opción viable de enseñanza-aprendizaje en matemática o de cualquier otra asignatura.

\section{Soportes de la Investiga- ción Ventajas del Modelo Escuela Nueva-Escuela Activa}

La Escuela Nueva Activa es considerada como un modelo pedagógico que fue diseñado en Colombia a mediados de los años setenta por Vicky Colbert, Beryl Levinger y Óscar Mogollón para ofrecer la primaria completa y mejorar la calidad y efectividad educativa en zonas de difícil acceso de las escuelas del país. Se focalizó inicialmente las escuelas rurales (Fundación Escuela Nueva, 2009, p. 44).

La metodológica cuenta una propuesta didáctica acompañada de cartillas con unidades y guías, las cuales desarrollan una secuencia didáctica; despliega políticas, planes y proyectos sociales. Este modelo educativo invita a innovar en la parte académica, orientar y facilitar el conocimiento, fomenta el aprendizaje participativo, colaborativo,
Las TIC se han convertido en herramientas lúdicas en el momento de afianzar los conocimientos, desarrollando actividades de observación, manipulación, clasificación, comparación, experimentación. 
La escuela nueva cuenta con cuatro componentes que interactúan entre sí, estos son: el componente curricular, que tiene como finalidad mostrar una serie de estrategias apropiadas a las necesidades del medio donde se aplique situado en el constructivismo. Promueve el enfoque multigrado, aprendizaje individual y colectivo, fortalecimiento de la relación escuela-comunidad para la adquisición de saberes; respeta ritmos de aprendizaje, la evaluación es continua; entrega un conjunto de principios pedagógicos y recursos en la práctica de aula; genera convivencia pacífica en ambientes democráticos y de convivencia participativa, de liderazgo, autonomía entre el docente-alumno; genera aprendizaje activo participativo; incluye material didáctico y mobiliario; desarrolla estrategias de aula a través de las guías de aprendizaje, los CRA (centros de recursos de aprendizaje) o rincones de aprendizaje, la biblioteca. El aprendizaje se da acorde a los intereses y necesidades.

La escuela nueva cuenta con cuatro componentes que interactúan entre sí, estos son: el componente curricular, que tiene como finalidad mostrar una serie de estrategias apropiadas a las necesidades del medio donde se aplique; el componente comunitario, cuya finalidad es fortalecer las relaciones escuela comunidad; el componente de formación para los docentes, que les permite capacitarse y estar actualizándose en beneficio de la labor académica; y un componente de gestión, que busca dar respuesta a las necesidades de los actores y de la institución. De ello, depende el éxito de la escuela nueva. Aspectos importantes de esta investigación (Fundación Escuela Nueva, 2009, p. 44).

Se puede establecer que la actividad práctica dentro de la clase, permite que los estudiantes consoliden el aprendizaje a través de la experiencia, la ejecución de trabajos que conlleven a desarrollar habilidades y destrezas para lograr un desempeño ágil y eficaz en un saber, integrando teoría y práctica, donde el estudiante actúa de acuerdo con los nuevos conocimientos, luego aplique lo aprendido en cualquier situación de su vida diaria, estimulándolo a que profundice, y recurra a diversas estrategias, busca solucionar problemas que se le presenten. Se motiva a la institución a utilizar software libres como estrategia didáctica en el aula para fortalecer procesos de aprendizaje (Fundación Escuela Nueva, 2009, p. 286).

\section{La enseñanza y el aprendizaje de las matemáticas}

La enseñanza se debe concebir como una actividad realizada conjuntamente mediante la interacción de tres elementos: un profesor o docente, uno o varios alumnos y el objeto de conocimiento (Díaz, 2001). El docente transmite sus conocimientos a los alumnos a través de diversos medios, técnicas y herramientas de apoyo; siendo él, la fuente del conocimiento; y el alumno, receptor.

Díaz (2001) afirma que si el aprendizaje es uno de los principales procesos a desarrollar en la escuela, la enseñanza también es un proceso importante y principal para el desarrollo de habilidades y conocimientos de los estudiantes. En este sentido, Díaz (1997) indica que: "La enseñanza puede considerarse como un proceso que facilita la transformación permanente 
del pensamiento, las actitudes y los comportamientos de los alumno/as" (p. 12).

Según Gómez (citado en Miranda, 2009), entre las principales causas del temor de las matemáticas está la falta de conciencia del profesor y el papel importante que desempeña en el salón de clases, su responsabilidad con respecto al estudiante y el reto que significa ser profesor. El principal comprometido en el éxito o fracaso de la enseñanza es el profesor, el encargado de regular y matizar la enseñanza, puesto que es el único que en la práctica educativa le corresponde señalar lo que está mal y cómo corregirlo. Si reflexiona su práctica docente y la convierte, puede facilitar el aprendizaje de las matemáticas.

Fernández (2007) afirma que antes de entrar en el proceso multiplicativo, previamente, los alumnos han debido aprender a contar de dos en dos, de tres en tres, de forma seriada, ascendente y descendente. En el desarrollo de la noción de multiplicación, deben también seguirse las tres etapas metodológicas; en la primera, debe haber un intenso trabajo con cantidades discretas, usando objetos diferentes, como tapillas, piedrecillas, canicas, semillas y otros, al igual que cantidades continuas, usando regletas, áreas cuadriculadas. En la segunda, realizan una representación usado dibujos de los objetos y la suma entre ellos; grafican la suma usando la recta numérica, que permite la asociación entre avanzar como adicionar, y retroceder como quitar. La última etapa es la abstracción o representación en el que solo se usa el lenguaje de signos y numerales para figurar las operaciones. Sumando siempre grupos de objetos, iguales, de dos en dos o tres en tres. Lo que nos llevó a buscar en el software scratch una estrategia para enseñar las tablas de multiplicar y contribuir a adquirir procesos multiplicativos.

Muñoz (2010), en su tesis "Estrategias didácticas para desarrollar el aprendizaje significativo de las tablas de multiplicar en niños del grado 3 - b de la Institución Educativa José Holguín Garcés Sede Ana María De Lloreda”, manifiesta la indisposición y apatía de los estudiantes para aprender las tablas de multiplicar, quienes las consideran difíciles de aprender. Durante esta investigación, se analiza que las tablas de multiplicar son parte indispensable del proceso académico, que si se aprenden, los alumnos pueden resolver problemas que requieran de suma, resta, multiplicación y división con éxito, mejorando el rendimiento académico.

Montessori (1915) dice que los recursos materiales son clave para el aprendizaje. Ella da libertad para que los estudiantes aprendan y se dé un progreso positivo, apoya el uso de materiales manipulativos, manifiesta que se debe favorecer la autoeducación donde el docente solo interviene creando un ambiente idóneo. Esto permite que los alumnos sean gestores de su aprendizaje, que puedan realizar actividades lúdico-pedagógicas que favorezcan el aprendizaje.

Finalmente, de acuerdo con Lucio (2007), se tiene en cuenta que la Escuela Nueva es una propuesta pedagógica,
Fernández (2007) afirma que antes de entrar en el proceso multiplicativo, previamente, los alumnos han debido aprender a contar de dos en dos, de tres en tres, de forma seriada, ascendente y descendente. 
y un modelo educativo dirigido a multigrados de las zonas rurales, en donde los niños de diferentes grados cuentan con un solo docente que orienta su proceso de aprendizaje, el cual necesita de diversas herramientas educativas para generar una buena educación. De acuerdo con la praxis, a un docente de escuela unitaria que orienta tres o más grupos a la vez, le es complicado dedicar tiempo suficiente para profundizar muchos ejes temáticos; si se utilizan herramientas didácticas, en este caso scratch, se puede dejar al alumno que aprenda las tablas de multiplicar en la actividad práctica y se dedica el tiempo a otro grupo para fortalecer el proceso de aprendizaje, generando tranquilidad al observar que el alumno está en actividades que le favorecen y que fortalece procesos académicos.

\section{El uso de las tecnologías de la información y comunicación en educación (TIC)}

El software scratch es una herramienta indispensable en la clase de computación de nivel básico, ya que los niños deben desarrollar un pensamiento algorítmico, creatividad y destreza para resolver problemas que vayan surgiendo durante el ciclo escolar, no solo en computación sino en cualquier materia e incluso problemas de la vida cotidiana (Marmolejo, 2012).
Según Escontrela y Stojanovic (2004), la importancia de una correcta apropiación de las TIC en los procesos de enseñanza-aprendizaje, tiene que estar integrada a las políticas educativas, asumiendo la importancia e impacto en la educación, haciendo énfasis en los problemas relacionados con el analfabetismo digital de algunos miembros de la comunidad educativa y la falta de infraestructura tecnológica que no permiten una apropiación completa para que puedan integrar las herramientas tecnológicas al modelo pedagógico, las instituciones educativas deben estar a la vanguardia de las Nuevas Tecnologías para poder capacitar a docentes y, en general, a la comunidad educativa para lograr mayor cobertura en el currículo y fortalecimiento de habilidades $y$ destrezas.

\section{El software Scratch en los procesos de aprendizaje}

El software scratch es una herramienta indispensable en la clase de computación de nivel básico, ya que los niños deben desarrollar un pensamiento algorítmico, creatividad y destreza para resolver problemas que vayan surgiendo durante el ciclo escolar, no solo en computación sino en cualquier materia e incluso problemas de la vida cotidiana (Marmolejo, 2012).

Desde el lanzamiento público en mayo de 2007, el sitio Web de Scratch (MIT, 2014) se ha convertido en una vibrante comunidad en línea, en donde sus miembros comparten, discuten y crean nuevas versiones de otros proyectos. Tal y como lo señala MIT (2014), Scratch ha sido llamado "el YouTube de los medios interactivos", ya que cada día, usuarios de Scratch o Scratchers de todo el mundo suben más de 1.500 nuevos proyectos al sitio, cuyo código fuente se encuentra disponible libremente para ser compartido con los demás usuarios. La colección de proyectos que se encuentra en este sitio es tremendamente diversa, incluyendo juegos de video, boletines de noticias interactivas, simulaciones científicas, visitas virtuales, tarjetas de 
cumpleaños, concursos de baile de animación, y tutoriales interactivos, todos ellos programados en Scratch (Vidal, 2015). Estos proyectos están relacionados con las diferentes áreas del conocimiento y se pueden aprovechar en el aula, como estrategia didáctica.

Al programar con Scratch y compartir los proyectos interactivos, se pueden aprender importantes conceptos matemáticos y computacionales, así como la forma de pensar creativamente, razonar sistemáticamente y trabajar en colaboración: todas ellas, habilidades esenciales para el siglo XXI (Delors, 2006).

\section{El juego como estrategia de aprendizaje}

La investigación nos lleva a tener en cuenta que el juego contribuye en situaciones que invita a la reflexión en el aprendizaje de la matemática; sin embargo, es importante que sea el profesor quien genere, participe y planifique juegos para los niños de educación primaria.

En cuanto al juego como estrategia para el aprendizaje de la matemática, este muestra dos aspectos diferenciados: uno formativo y otro informativo. El primero está orientado a establecer una motivación y una experiencia directa en el estudiante por el uso del juego como un medio significativo para el aprendizaje de la matemática; despliega procesos motivacionales y de un hacer con sentido al ser actor principal en el proceso de aprender, el cual tendrá que poner en práctica cuando se enfrente a situaciones problemáticas.
De acuerdo con Vidal (2014), en cuanto al aspecto informativo, agrega conocimientos, habilidades y destrezas con relaciónaloscontenidos matemáticos, sobre todo en la planificación y ejecución de algoritmos que admitan el desarrollo de capacidades en los niños y niñas. Ambos aspectos son desarrollados en la experiencia con Scratch, ya que este lenguaje de programación permite definir objetos gráficos con propiedades y acciones definidas, crear secuencia de interacción entre dichos objetos, esto es, definir algoritmos sobre dichos objetos, cuyos efectos son visibles sobre los objetos. Por este efecto de acciónreacción en los algoritmos definidos sobre objetos gráficos en Scratch, los estudiantes se motivan al ver de forma real lo que ellos pueden hacer sobre los objetos de soluciones algorítmicas, además de incorporar, muchas veces por descubrimiento, una serie de contenidos o conocimiento en su aprendizaje. Durante los últimos cinco años, la institución ha logrado grandes éxitos con el software scratch, del cual sale esta propuesta educativa para buscar soluciones académicas y buen uso del tiempo libre.

\section{Metodología}

El presente trabajo de investigación se fundamentó teniendo en cuenta el problema de investigación, el cual se abordó desde un paradigma críticosocial a partir de un enfoque cualitativo. De esta manera, el diseño del proyecto se basó en la investigación acción (IA), teniendo en cuenta que este tipo de investigación interpreta lo que ocurre desde el punto de vista de quienes actúan e interactúan en la situación
La investigación nos lleva a tener en cuenta que el juego contribuye en situaciones que invita a la reflexión en el aprendizaje de la matemática 
La investigación se realizó a partir de la observación y reflexión de la experiencia de prácticas matemáticas mediante el uso del scratch. problema, por ejemplo, profesores y alumnos, profesores y director. Según lo menciona Torrecilla (2010), se presenta como una metodología de investigación orientada hacia el cambio educativo y se caracteriza, entre otras cuestiones, por ser un proceso que, como señala Herreras (2004) en sus escritos, se construye desde y para la práctica, teniendo en cuenta cómo se debe llevar a cabo la docencia desde la investigación acción.

El tipo de enfoque fue mixto, por ello se usaron datos cuantitativos y cualitativos. De acuerdo con Hernández (2011), los métodos mixtos son una estrategia de investigación con la cual el investigador recolecta, analiza $\mathrm{y}$ mezcla, integrando y conectando tanto los datos cuantitativos como los cualitativos. Los métodos mixtos llevan a una mayor amplitud, profundidad, diversidad, riqueza interpretativa y sentido de comprensión de los datos.

Este tipo de enfoque se realizó con la intención de indagar y transformar las actuales prácticas pedagógicas respecto de la multiplicación de números enteros, así como la búsqueda de las mejores alternativas de abordaje y solución. Esta propuesta opta por el enfoque cualitativo, aplicado a la educación. Se resalta que se adoptó este enfoque, por ser flexible y abierto a toda clase de instrumentos y estrategias que promuevan la comprensión, la interpretación y valoración crítica de las concepciones que sustentan las prácticas, así como su relación e implicaciones con los niños (Cerda, 1991).
La investigación se realizó a partir de la observación y reflexión de la experiencia de prácticas matemáticas mediante el uso del scratch. En el modelo de Investigación Acción Educativa se encuentra al comienzo y al final del ciclo, en la planeación y en el seguimiento de la acción instaurada para transformar la práctica.

De esta manera, el objetivo es mejorar a través de su transformación y, al mismo tiempo que procura comprenderla, también demanda la participación de los sujetos en la mejora de sus propias prácticas, exige una actuación grupal por la que los sujetos implicados colaboran coordinadamente en todas las fases del proceso de investigación, realiza análisis crítico de las situaciones y se configura como una espiral de ciclos de planificación, acción, observación y reflexión; de esta manera, se realizó el proceso para el diseño y la implementación de la herramienta didáctica. El enfoque metodológico del proyecto, está basado en lo propuesto por Kemmis (1998).

\section{Instrumentos para la recolección de información}

Para hacer el análisis de la investigación, se utilizaron como instrumentos una encuesta, este se aplicó a siete estudiantes para determinar su gusto por las tablas de multiplicar, la metodología de la enseñanza de matemáticas por parte del docente y el gusto por el uso de las TIC; una entrevista a seis docentes, para determinar la metodología aplicada a la hora de enseñar las tablas de multiplicar, determinar las dificultades 
que presentan los estudiantes, estrategias y formas de evaluar.

Se realizó una prueba de entrada salida. Se realizó para establecer el conocimiento de los estudiantes y el manejo de las tablas de multiplicar.

Además, se utilizó un diario de campo, donde se registró, sistematizó y validó, de manera confiable, los comportamientos o conductas manifestadas por los sujetos de la investigación. Este puede servir para determinar la aceptación de un grupo respecto a su profesor, analizar conflictos dentro del aula, relaciones entre pares, y avances de los estudiantes.

Se fijaron unas fases que partieron de la observación, que permitió reconocer los problemas que existen en los estudiantes al momento de realizar las multiplicaciones, identificando debilidades y fortalezas; luego, una planificación, en este aparte se diseñó y realizó un plan de acción, para la implementación de actividades microcurriculares utilizando scratch para apoyar la enseñanza de las tablas de multiplicar de números naturales; y, posteriormente, una fase de ejecución o acción, en esta etapa se aplicaron las actividades diseñadas en scratch, mediante el uso de computadores. Finalmente, una fase de reflexión por parte del estudiante sobre los pasos aplicados en la resolución de una tarea, de manera que este trabaje sus destrezas metacognitivas.

Después de evaluar la estrategia didáctica y su impacto en la población, se evalúa para determinar fortalezas y debilidades. Para ello, se realizó un diagnóstico para identificar las debilidades y fortalezas de los estudiantes.

Esto permitió hacer un análisis categórico, teniendo en cuenta la actitud del estudiante, en la parte de motivación e interés. En el desarrollo lógico matemático, se tuvo en cuenta la realización de un ejercicio para identificar la capacidad de abstracción en el desarrollo de ejercicios de multiplicación, luego se hizo el análisis de la información que aparece a continuación.

Esto permitió diseñar las actividades acorde al uso del software scratch, siguiendo primero un instructivo del uso del software scratch, se enseñaron las características de este, las herramientas que tiene, su uso y se dio paso a la manipulación libremente de la estrategia didáctica. Los estudiantes se apropiaron de los diferentes programas manifestando interés y ánimo al realizar las operaciones solicitadas. En esta actividad, detectamos que a los estudiantes les llamó la atención la parte multimedia: imagen, sonido, interactividad, movimiento. Esta fue una señal de agrado y aceptación hacia la estrategia usada.

Posteriormente, se realizaron dos estrategias, la primera se llevó a cabo con la metodología escuela nueva y la praxis para observar qué actividades incluir para fortalecer procesos multiplicativos; y, a la par, la segunda se realizó teniendo en cuenta la praxis en TIC por el otro investigador, quien buscó las actividades que se acogieran a las edades de los estudiantes para
Se fijaron unas fases que partieron de la observación, que permitió reconocer los problemas que existen en los estudiantes al momento de realizar las multiplicaciones, identificando debilidades y fortalezas 
Tabla 1. Análisis categorías estudiantes y docentes

\begin{tabular}{|c|c|c|c|}
\hline & Categorías & Antes de la propuesta & Después de la propuesta \\
\hline \multirow[t]{2}{*}{$\begin{array}{l}\text { Actitud del } \\
\text { estudiante }\end{array}$} & Motivación & $\begin{array}{l}\text { Poco agrado por } \\
\text { la realización de } \\
\text { multiplicaciones. }\end{array}$ & $\begin{array}{l}\text { Las actitudes mostradas por los estudiantes fueron positivas } \\
\text { en cuanto a estado de ánimo, entusiasmo, agrado, optimismo, } \\
\text { interacción, comunicación, alegría, respeto, tolerancia y } \\
\text { optimismo en el desarrollo de actividades. } \\
\text { Se pudo captar el entusiasmo con que los estudiantes acometen } \\
\text { participativamente todas y cada una de las actividades, } \\
\text { poniendo en ellas toda su capacidad de acción en aras de } \\
\text { alcanzar los objetivos trazados, logro a logro y desarrollando } \\
\text { las competencias requeridas para tal fin, modificando su forma } \\
\text { de aprender. }\end{array}$ \\
\hline & Interés & $\begin{array}{l}\text { La posición asumida por } \\
\text { los estudiantes frente } \\
\text { a las asignaturas era de } \\
\text { apatía. }\end{array}$ & $\begin{array}{l}\text { La posición asumida por los estudiantes frente al reto de } \\
\text { aprender mediante el uso de scratch, fue activa ya que se } \\
\text { involucraron componentes cognitivos, afectivos, conductuales, } \\
\text { la capacidad creativa e ingenio para afrontar, formular y resolver } \\
\text { situaciones problémicas de multiplicación. }\end{array}$ \\
\hline \multirow[t]{4}{*}{$\begin{array}{l}\text { Desarrollo } \\
\text { lógico } \\
\text { matemático }\end{array}$} & $\begin{array}{l}\text { Realización de } \\
\text { ejercicios }\end{array}$ & $\begin{array}{l}\text { Los estudiantes no } \\
\text { realizaban los ejercicios } \\
\text { propuestos. }\end{array}$ & $\begin{array}{l}\text { Los estudiantes manifestaron su interés para interpretar, } \\
\text { comprender, argumentar, plantear y formular sus propios } \\
\text { procedimientos, tendientes a resolver situaciones problémicas } \\
\text { de multiplicación. }\end{array}$ \\
\hline & Abstracción & $\begin{array}{l}\text { Los estudiantes están } \\
\text { acostumbrados a } \\
\text { realizar los ejercicios } \\
\text { de forma repetitiva sin } \\
\text { entenderlos. }\end{array}$ & $\begin{array}{l}\text { Los estudiantes dejan ver su capacidad para ir de la totalidad a } \\
\text { las partes, imaginar, deducir, descomponer, analizar y sintetizar, } \\
\text { respecto de situaciones problémicas de multiplicación. }\end{array}$ \\
\hline & Creatividad & $\begin{array}{l}\text { No existe la creatividad } \\
\text { en los estudiantes para } \\
\text { desarrollar o plantear } \\
\text { otras maneras de resolver } \\
\text { multiplicaciones. }\end{array}$ & $\begin{array}{l}\text { Los estudiantes demuestran capacidad mediante sus } \\
\text { competencias para reconocer saberes, ejecutar proyectos y } \\
\text { procesos; visión futurista e imaginación y síntesis. }\end{array}$ \\
\hline & Razonamiento & Mecanizado. & $\begin{array}{l}\text { Predicciones y dar posibles respuestas a situaciones problémicas } \\
\text { de tipo multiplicativo, evidenciando el desarrollo lógico } \\
\text { matemático en los estudiantes que se manifestó en la forma de } \\
\text { pensar, reflexionar, dar a conocer sus puntos de vista, establecer } \\
\text { relaciones causa-efecto, plantear hipótesis, hacer aproximaciones. }\end{array}$ \\
\hline
\end{tabular}

Fuente: elaboración propia, 2016 primer trimestre 2017.

fortalecer estos ejes temáticos a través de proyectos en scratch, y se adapten a estos ejes temáticos relacionados con las tablas de multiplicar; luego, se diseñaron las actividades faltantes en el software; posteriormente, se realizó la parte práctica, donde se interactuó, escogiendo las más indicadas, para este fin. Durante la parte práctica, se llevó un diario de campo donde se registraron las experiencias, también se entrevistó a cada estudiante para obtener información de primera mano.

Es importante destacar de la actitud participativa, el entusiasmo y el agrado manifestado por el colectivo de estudiantes ante la sencillez y 
variedad de las actividades propuestas y ejecutadas de manera clara, eficaz y efectiva, en procura de la producción de nuevo conocimiento y a la comprensión de la conceptualización.

Después de haber formalizado y haber trabajado ejercicios, se verifica el aprendizajedelosestudiantes:seobservan mejoras referidas a las habilidades cognitivas de carácter operacional, habilidades cognitivas de carácter conceptual, habilidades metacognitivas al establecer analogías equivalentes en el proceso multiplicativo; cualidades de los alumnos: al desarrollar actitudes positivas hacia la multiplicación; solución de operaciones, al realizar la prueba de salida se observa cómo se disminuye notablemente el número de errores a la hora de multiplicar y realizar ejercicios que requieren de la multiplicación.

A continuación, se presenta el análisis de las actividades (ver tabla 2).

Tabla 2. Análisis actividades

\begin{tabular}{|c|c|}
\hline Textual & Análisis de la información \\
\hline $\begin{array}{l}\text { Dificultad en el pensamiento } \\
\text { matemático }\end{array}$ & $\begin{array}{l}\text { Al comienzo, se presentó dificultad en el manejo de algunos botones de scratch. } \\
\text { Debido a que se da prioridad a procesos mecánicos, los estudiantes tienen algún grado } \\
\text { de dificultad, pero con las actividades se apropian de la manera de ejercitar las tablas de } \\
\text { multiplicar. } \\
\text { La aplicación de conceptos, tanto matemáticos como de informática, dependen de la } \\
\text { didáctica que se usa. } \\
\text { Los estudiantes tienen dificultad en expresar conceptos con sus propias palabras. }\end{array}$ \\
\hline $\begin{array}{l}\text { Gusto por las tablas de } \\
\text { multiplicar }\end{array}$ & $\begin{array}{l}\text { Se evidencia el interés de los estudiantes en la realización de las actividades: } \\
\text { - Realizan las actividades con gran entusiasmo e interés, al igual que se muestran inquietos } \\
\text { por saber qué más actividades hay. } \\
\text { - El desempeño se hace más eficiente y avanza más rápidamente en la comprensión de los } \\
\text { conceptos. } \\
\text { Los estudiantes muestran interés en el manejo del scratch. } \\
\text { La clase se hace más dinámica, los estudiantes aprenden mientras exploran scratch. } \\
\text { Los estudiantes interpretan y distinguen diferentes maneras de aprender las tablas. } \\
\text { El uso de las TIC (scratch) permite una mejor recreación de los problemas y además } \\
\text { permiten las posibles soluciones. }\end{array}$ \\
\hline $\begin{array}{l}\text { Incidencia de la metodología } \\
\text { del profesor }\end{array}$ & $\begin{array}{l}\text { La metodología presenta oportunidades de motivación de los estudiantes. Los estudiantes } \\
\text { permanecen a la expectativa de la siguiente actividad. } \\
\text { Los docentes, en general, tienden a utilizar la práctica tradicional en sus clases ya que se da } \\
\text { más importancia a la mecanización de procesos y no al manejo y aplicación de conceptos. }\end{array}$ \\
\hline $\begin{array}{l}\text { Favorece el horario según la } \\
\text { distribución en la áreas }\end{array}$ & $\begin{array}{l}\text { Favorece el horario, ya que los estudiantes realizan los trabajos con gran agilidad. } \\
\text { Los estudiantes se mostraron muy activos y dinámicos, ya que presentan facilidad para el } \\
\text { manejo de los computadores. } \\
\text { No hay distractores externos y esto permite que los estudiantes obtengan un mayor } \\
\text { aprendizaje significativo. }\end{array}$ \\
\hline
\end{tabular}




\begin{tabular}{|l|l|}
\hline Actitud & $\begin{array}{l}\text { Los estudiantes cambiaron de actitud, pasaron de ser receptores a ser protagonistas en las } \\
\text { actividades a realizar. }\end{array}$ \\
\hline Avances & $\begin{array}{l}\text { Los estudiantes construyeron y asimilaron conceptualizaciones y lograron un aprendizaje } \\
\text { colaborativo y cooperativo. Los estudiantes se mostraron interesados en realizar la } \\
\text { actividad y la resolvieron haciendo aportes personales y en forma grupal. Se destaca la } \\
\text { actitud participativa y el agrado manifestado ante la actividad propuesta. } \\
\text { Los estudiantes intercambiaron información y crearon un lenguaje formal y adecuado, } \\
\text { para explicar los procedimientos a seguir para reconocer los procesos de multiplicar. La } \\
\text { actitud de los estudiantes fue positiva y, con creatividad, resolvieron las actividades. } \\
\text { Se motivan para trabajar con computadores. }\end{array}$ \\
$\begin{array}{l}\text { El estudiante participa activamente en este tipo de actividades, ya que se pierde la monotonía } \\
\text { de las clases magistrales. } \\
\text { Al usar scratch, se contribuyó de manera directa con el uso adecuado de las nuevas } \\
\text { tecnologías, permitiendo además el desarrollo de habilidades de pensamiento. } \\
\text { Cada estudiante protagonizó su proceso de aprendizaje mediante la utilización de scratch, } \\
\text { se superaron las dificultades presentadas en los contenidos mediante la clase tradicional, en } \\
\text { la medida que iban desarrollando habilidades y destrezas para utilizar un conocimiento y } \\
\text { la forma de aplicarlo creativamente desde un racionamiento lógico. }\end{array}$ \\
\hline
\end{tabular}

Como soporte de la investigación, también se llevó a cabo la triangulación.

De acuerdo con la triangulación, se observó que las actividades con scratch promovieron la autonomía, creatividad, sentido crítico de los estudiantes; se les inculcó el sentido de compromiso y responsabilidad para consigo mismo y para con los compañeros, toda vez que aprenden a tomar decisiones tendientes a la solución de actividades planteadas.

Los estudiantes manifestaron gusto por la forma de trabajo en clase. Las actividades propuestas les permitieron pensar y analizar, haciendo uso de elementos cognitivos, y ayudando a la construcción de conceptos. Igualmente, los estudiantes se concientizaron de la importancia del desarrollo de habilidades matemáticas.
Los estudiantes realizan la composición, descomposición y representación de cantidades, resolvieron problemas en situaciones multiplicativas, razonaron adecuadamente para entender y enfrentar problemas retadores, se reconocieron características de los números y manejar sus operaciones.

\section{Impacto}

Los estudiantes, al entrevistarlos, dicen que las clases fueron amenas, divertidas, les gustó el uso de scratch, aprenden mejor y distinto, la clase es divertida, motiva a aprender y trabajan diferentes temas. De acuerdo con lo anterior, se puede deducir que al usar scratch para fortalecer el aprendizaje de las tablas de multiplicar, permitió a los estudiantes potenciar habilidades matemáticas $\mathrm{y}$ 


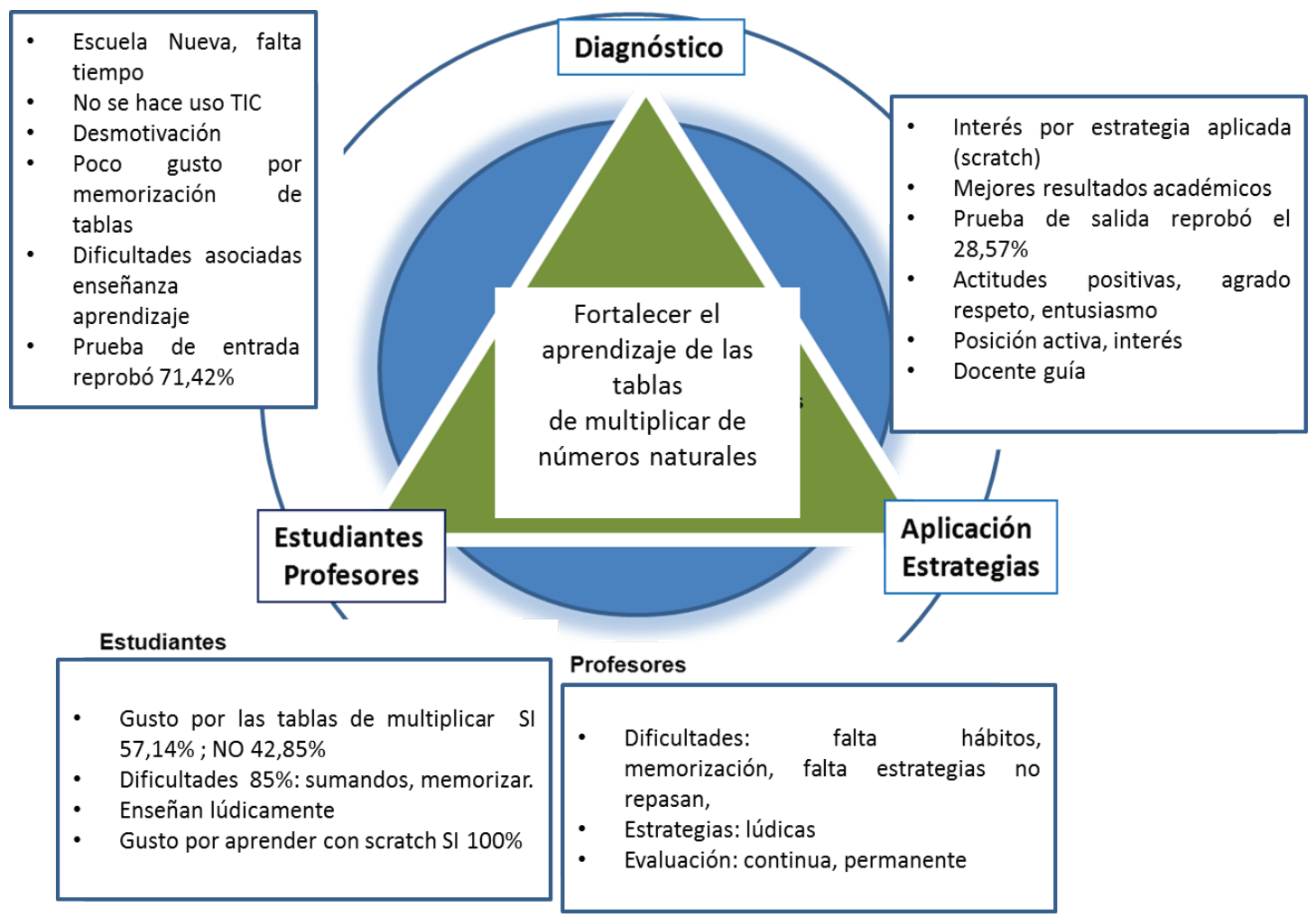

Figura 20. Triangulación.

Fuente: elaboración propia.

se evidenció una positiva incidencia frente al aprendizaje.

Se comprobó que sí es posible romper esquemas tradicionales de aprendizajes mediante el uso de las TIC, en este caso scratch, para desarrollar habilidades de pensamiento matemático y mejorar la motivación frente al aprendizaje de las tablas de multiplicar.

Se logró que los estudiantes cambiaran su actitud negativa hacia el aprendizaje de las tablas de multiplicar. Con el desarrollo de las actividades, se crearon clases dinámicas y activas que lograron incentivar el deseo de aprender de los estudiantes; y estas experiencias vividas al ser evaluadas, permitieron evidenciar resultados positivos que en la actualidad son compartidos con los demás docentes y padres de familia quienes resaltaron el cambio de actitud de sus hijos respecto a las tablas de multiplicar, su responsabilidad y gusto por aprender haciendo uso de Scratch. El desarrollo de este trabajo permitió compartir experiencias con otros docentes de diferentes instituciones educativas, quienes reconocieron la funcionalidad del uso de las TIC para el aprendizaje de las tablas de multiplicar. 
Tabla 3. Impacto docentes.

\begin{tabular}{|c|c|}
\hline ÍTEM & Respuesta de los estudiantes \\
\hline Eficacia & $\begin{array}{l}\text { Las actividades ejecutadas a lo largo de la investigación, se analizaron desde la perspectiva } \\
\text { de las categorías establecidas, permitiendo la observación, apreciación y percepción de } \\
\text { procesos mentales cognitivos. }\end{array}$ \\
\hline Efectividad & $\begin{array}{l}\text { Los estudiantes fortalecieron el aprendizaje de las tablas de multiplicar, formándose } \\
\text { mediante la innovación y no el memorizar. } \\
\text { Se promovieron procesos de reflexión y análisis. }\end{array}$ \\
\hline Apropiación & $\begin{array}{l}\text { Los estudiantes valoran que se cambie la clase magistral y la manera de presentar los } \\
\text { contenidos. De esta manera, se obtienen mejores resultados. } \\
\text { Se logró que los estudiantes fueran protagonistas de su propia enseñanza haciendo crecer } \\
\text { su creatividad y activar sus cualidades. }\end{array}$ \\
\hline $\begin{array}{l}\text { Debilidades, } \\
\text { fortalezas y } \\
\text { facilidades }\end{array}$ & $\begin{array}{l}\text { Todo docente debe estar continuamente capacitándose, de esta manera vive actualizado } \\
\text { y puede proponer cambios en su manera de enseñar desde el ámbito de las nuevas } \\
\text { tecnologías de la información. } \\
\text { Es importante que los docentes cambien las clases magistrales para que los estudiantes se } \\
\text { motiven. Existe la necesidad de motivar, incentivar, proponer y aplicar una planificación } \\
\text { del aprendizaje del álgebra que ponga al servicio de todos los estudiantes, estrategias } \\
\text { metodológicas, didácticas y evaluativas que les permitan afrontar situaciones problémicas } \\
\text { de su vida cotidiana, con las herramientas necesarias para darles solución de manera clara, } \\
\text { coherente y precisa, obteniendo con ello un apropiado desarrollo del pensamiento lógico } \\
\text { matemático. } \\
\text { Ser docente de matemáticas constituye un verdadero reto por el dominio de los contenidos } \\
\text { y poder llegar adecuadamente a los estudiantes. Es necesario el dominio de la didáctica } \\
\text { en general, las metodologías, estrategias y formas para compartir las experiencias de } \\
\text { aprendizaje con los estudiantes. }\end{array}$ \\
\hline Avances & $\begin{array}{l}\text { No todos los estudiantes responden de la misma manera a las estrategias usadas. Aquí } \\
\text { se plantean algunas diferencias: temores, baja comprensión, presión del grupo, falta de } \\
\text { interés, desconocimiento de conceptos. }\end{array}$ \\
\hline
\end{tabular}

Fuente: elaboración propia.

\section{Discusión a la luz de los autores}

Este trabajo se fundamentó en el constructivismo de Piaget (1998) y el construccionismo de Papert (1987), con respecto al aprendizaje como proceso donde las personas participan activamente en la construcción de su propio conocimiento a partir de experiencias como el uso de scratch, que se constituyó como herramienta apropiada para el desarrollo y fortalecimiento de habilidades de pensamiento matemático para mejorar las competencias matemáticas, aplicada a las tablas de multiplicar.

Ahora bien, de acuerdo con Piaget (1998), el sujeto construye conocimiento a medida que interactúa con la realidad. Este es un proceso de interacción sujeto-objeto, por medio de una acción transformadora pasando de un estado de menor conocimiento a otro de mayor conocimiento, que permite surgir una nueva estructura mental. En el caso de la enseñanza de 
matemáticas, se pasa de la teoría que facilita la intervención en procesos de enseñanza y aprendizaje por medio del constructivismo actuando como papel integrador.

Es de anotar que, de acuerdo con Piaget, el sujeto construye su conocimiento a medida que interactúa con la realidad; ahí se produce un proceso de interacción sujeto-objeto por medio de una acción transformadora en donde los estudiantes reestructuran sus esquemas cognitivos.

Se está de acuerdo con Monereo (1998), quien dice que la enseñanza puede considerarse como un proceso que facilita la transformación permanente del pensamiento, las actitudes y los comportamientos de los alumno/as.

Ahora bien, teniendo en cuenta lo fácil que resulta el manejo de la tecnología para los estudiantes, se convirtió en fortaleza y oportunidad para llevar el conocimiento de manera amigable, flexible y amena, para lograr un aprendizaje útil y oportuno como una ayuda en el aula que fortalece los rincones de aprendizaje.

Es importante resaltar, de acuerdo con Echeverría (2001), que al hacer uso de la tecnología es necesario tener en cuenta varias condiciones para tener éxito, entre las cuales se pueden resaltar el conocimiento de los docentes en el uso de las TIC, los estudiantes deben tener conocimiento en el manejo de la información, eliminar exigencias de espacio y tiempo. Se observó que los estudiantes manejaron la información conduciéndola hacia la práctica del autoaprendizaje, con el fin de que fuera significativo.

De igual manera, se está de acuerdo con Papert (1987) quien considera que las TIC no son solo una oportunidad sino también la excusa perfecta para introducir en la educación nuevos elementos que realizan una gran transformación en la práctica educativa, así se amplía la gama de recursos didácticos, estrategias pedagógicas, superando de esta manera la enseñanza basada en transmisión de contenidos y se desarrollan habilidades para el aprendizaje activo donde los estudiantes son protagonistas de su aprendizaje activo y constructivo.

\section{Impacto institucional}

La experiencia didáctica presentada contribuye al enriquecimiento del modelo pedagógico de la Institución, estableciéndose como plan de mejoramiento y seguimiento académico de la gestión de aula y diseño curricular, para el área de matemáticas.

Es de anotar que, Scratch puede ser usado en otras áreas del conocimiento y partiendo de situaciones o temas construidos en el aula favorecen el desarrollo de competencias comunicativas llevando al estudiante a narrar, graficar, animar, proponer, crear soluciones y representarlas. 


\section{Referencias bibliográficas}

Bernal, C. (2009). Metodología de la Investigación Cientifica. Bogotá: Pearson, Prentice Hall.

Burbules, N., \& CAllister, T. (2001). Educación: riesgos y promesas de las nuevas tecnologías de la información. Barcelona: Granica.

CÁRDENAS, R. (2010). Fortalecimiento del proceso de aprendizaje de la multiplicación a través de la lúdica. Tunja: Universidad Pedagógica y Tecnológica de Colombia.

Castro Bayona, O.L., \& Rincón Alfonso, R. E. (2015). Diseño de un material educativo computarizado para facilitar el aprendizaje de las tablas de multiplicar en el colegio Boyacá de Duitama. Tesis de Maestría. Bucaramanga, Colombia. Universidad de Santander.

Campo SaAvedra, M. F. (2013). Competencias TIC para el desarrollo profesional docente. Oficina de Innovación Educativa con Uso de Nuevas Tecnologías.

Cerda, H. (1991). Los elementos de la investigación. Bogotá: El Búho.

De Guzmán, O. M. (1993). Enseñanza de las ciencias y la matemática. España: Ed. Popular.

Delors, J. (2006). La Educación Encierra un Tesoro. Santillana, Madrid: Ediciones Unesco. Madrid.

DíAz, B. (2001). Estrategias Docentes para un aprendizaje significativo: una interpretación constructivista, (2a ed.). México: McGraw-Hill.

Díaz Barriga, F., \& Hernández, G. (1997). Estrategias docentes para un aprendizaje significativo. Una interpretación constructivista. México: McGraw-Hill.

Echeverría, J. (2001). Las TIC en educación. Revista Iberoamericana, 6 (24 pág.), $1-28$.

Escontrela Mao, R., \& Stojanovic Casas, L. (2004). La integración de las TIC en la educación: Apuntes para un modelo pedagógico pertinente. Revista de Pedagogía, 25(74), 481-502. Recuperado el 21 de febrero de 2017, de http://www.scielo. org.ve/scielo.php?script=sci_arttext\&pid=S0798-97922004000300006\&lng=e s\&tlng=es.

Fernández Bravo, J. (2007). La Enseñanza de la Multiplicación Aritmética: Una Barrera Epistemológica. Revista Iberoamericana de Educación, (43), 119-130.

Flórez, M., \& Rodríguez, L. (2009). El juego como estrategia lúdica para entender las tablas de multiplicar. Florencia: Centro Educativo Vanegas Florencia Caqueta.

Fundación Escuela Nueva. (2009). Escuela nueva - escuela viva, Volvamos a la gente, Manual para el docente. Bogotá: Quebecor Bogotá S.A.

García Ortega, N. (2016). Motivación en el aprendizaje de la programación a nivel bachillerato utilizando un lenguaje de programación educativo. (Tesis de maestría). Universidad Autónoma de Aguascalientes, Aguascalientes, México.

Hernández Sampieri, R., Fernández Collado, C., \& Baptista Lucio, P. (2011). Metodología de la Investigación. Mexico. Editorial McGraw-Hill. 
Herreras, E. B. (2004). La docencia a través de la investigación-acción. Revista Iberoamericana de Educación, (20), 7-39. Recuperado de http://www.rieoei.org/ deloslectores/682Bausela.PDF.

IAFRANCESCO, G. (2003). La investigación en Educación y pedagogía: Fundamentos y técnicas. Bogotá: Cooperativa Editorial Magisterio.

IsodA, M., \& Olfos, R. (2009). El Estudio de Clases y las demandas curriculares, La enseñanza de la multiplicación.

Jonassen, D. (1996). Los computadores como herramienta de la mente. TechTrends, 43(2), 24-32. Recuperado de http://www.aect.org/Intranet/Publications/ index.html.

Leguizamón González, M.C. (2004). Diseño y desarrollo de materiales educativos computarizados (MEC's): una posibilidad para integrar la informática con las demás áreas del currículo. Ponencia llevada en Aprendizaje y Currículo. Universidad Pedagógica y Tecnológica de Colombia, Tunja, Colombia.http:// www.colombiaaprende.edu.co/html/mediateca/1607/articles-106492_ archivo.pdf.

Lucio, R. (2007). La Escuela Nueva y los estándares académicos. Análisis y sugerencias. Bogotá: Documento elaborado para el MEN.

Macintosh. (1929). En: Resolución 2343 de 1994, del MEN. Santa Fe de Bogotá.

Marín GutiérRez, S. (2013). El entorno virtual de scratch como mediación lúdico pedagógica para potenciar la comprensión del plano cartesiano. (Tesis de maestría). Universidad Nacional de Colombia, Manizales, Colombia.

Marmolejo Valle, J. (2012). Pensamiento lógico matemático con scratch en nivel básico. Revista Vínculos, 9(1), 87-95. Recuperado de http://revistas.udistrital. edu.co/ojs/index.php/vinculos/article/view/4208/5865

MÉndez, E. (2010). Planificación para el aprendiaje de la multiplicación y la división de números naturales en el grado sexto. Tunja: Universidad Pedagógica y Tecnológica de Colombia.

Mendoza Ortiz, C. (2015). Scratch y G compris herramientas didácticas en el proceso de aprendizaje de las tablas de multiplicar en los estudiantes del grado $3^{\circ}$ de la Institución Educativa Rio Blanco sede G Alto Ceiba municipio del Peñón Santander. Tunja: Universidad Pedagógica y Tecnológica de Colombia.

Miranda Arroyo, J. C. (2009). Producción de estrategias de conteo para solucionar problemas de tipo aditivo y sustractivo en preescolares. Revista electrónica de didáctica de las Matemáticas, 3(3), 105-116.

MIT. (2014). Media Lab MIT, Ayuda sobre Scratch - Acerca de Scratch (en línea), 2003. Recuperado el 29 de junio de 2014, de http://scratch.mit.edu/about/.

Monereo, C., \& CAstelle, M. (1998). Las estrategias de aprendizaje: Cómo Incorporarlas a la práctica educativa. Barcelona: Edebc.

Montessori, M. (1915). Autoeducación. Pedagogía científica. Barcelona: Araluce. 
MuÑoz, C. (2010). Estrategias didácticas para desarrollar el aprendizaje significativo de las tablas de multiplicar en niños del grado $3-b$ de la Institución Educativa José Holguin Garces - Sede Ana María De Lloreda. (Tesis de especialización). Universidad de La Sabana, Bogotá, Colombia.

Papert, S. (1987). Desafío de la mente (5a ed.). Buenos Aires, Argentina: Ediciones.

Piaget, J. (1998). La construcción de lo real en el niño. El desarrollo de la noción de objeto. Argentina: Editorial Nueva Visión.

Resnick, M., Maloney, J., Monroy-Hernández, A., Rusk, N., Eastmond, E., Brennan, K. et al. (2009). Scratch: Programming for All. Communication of the ACM, 52(11), 60, 67.

Resolución 2343 de 1994 (Lineamientos Curriculares). MEN. Santa Fe de Bogotá.

Rico, L. (2007). La competencia matemática en PISA. PNA, 1(2), 47-66. Recuperado de http://funes.uniandes.edu.co/529/1/RicoL07-2777.PDF.

Segarra, L. (2002). Juego y Matemáticas. La Resolución de Problemas en Matemáticas. Caracas: Laboratorio Educativo.

Torrecilla, J M. (2010). Investigación acción. Métodos de investigación en Educación Especial. 3a Educación Especial. Curso, 2011. (pp. 14-16).

Vidal, C. L., Cabezas, C., Parra, J. H., \& López, L. P. (2015). Experiencias Prácticas con el Uso del Lenguaje de Programación Scratch para Desarrollar el Pensamiento Algorítmico de Estudiantes en Chile. Formación universitaria, 8(4), 23-32. Recuperado de https://dx.doi.org/10.4067/S071850062015000400004. 